\title{
Chirality violating condensates in QCD and their connection with zero mode solutions of quark Dirac equations
}

\author{
B.L.Ioffe \\ Alikhanov Institute of Theoretical and Experimental Physics, \\ B.Cheremushkinskaya 25, 117218, Moscow, Russia
}

\begin{abstract}
It is demonstrated, that chirality violating condensates in massless QCD arise entirely from zero mode solutions of Dirac equations in arbitrary gluon fields. The model is suggested, where the zero mode solutions are the ones for quarks, moving in the instanton field. Basing on this model were calculated the quark condensate magnetic susceptibilities of dimensions $3(\chi)$ and $5(\kappa$ and $\xi)$. The good considence of the values $\chi, \kappa$ and $\xi$, obtained in this approach with ones, found from the hadronic spectrum ia a serious argument in favour, that instantons are the only source of chirality violating condensates in QCD. The temperature dependence of the quark condensate is discussed. It is shown that the phase transition, corresponding to the $T$-dependence of the quark condensate $\alpha(T)$ as an order parameter, is of the type of crossover.
\end{abstract}

Keywords: zero mode, instanton, condensat

12.38. Aw, 11.30. Rd, 11.15. Tk

It is well known, that because of small values of light quark masses the perturbative QCD pocesses the property of chiral symmetry in cases, when the heavy quarks contributions can be neglected. However, in the real hadronic world the chiral symmetry is badly violated. This statement evidently follows from the existence of large proton mass and the absence of the negative parity baryon, degenerate with proton. It is also well known that large values of light quark condensates indicate the violation of chiral symmetry in QCD. These two facts are deeply interconnected: the values of the proton mass can be expressed through the value of quark condensate [1].

In this talk I discuss the appearance of quark condensate in nonperturbative QCD. It is established the connection of quark condensate value with zero mode solutions of quark Dirac equation [2]. In this way it is clarified the nature of proton mass and, as a consequence, the nature of all visible mass in the Universe [3].

Consider QCD action in Euclidean space-time

$$
S=\frac{1}{4} \int d^{4} x G_{\mu \nu}^{2}-\int d^{4} x \sum_{f}\left[\psi_{f}^{+}\left(i \gamma_{\mu} \nabla_{\mu}+i m_{f}\right) \psi_{f}\right]
$$

where $G_{\mu \nu}^{n}$ is gluon field tensor, the sum is over quark flavours. 


$$
\nabla_{\mu}=\partial_{\mu}+i g \frac{\lambda^{n}}{2} A_{\mu}^{n}
$$

and $A_{\mu}^{n}$ is the gluon field. Pay attention, that in Euclidean formulation of QCD $\bar{\psi}$ is replaced by $\psi^{+}$. (The review of Euclidean formulation of QCD and instantons is given in [4], see especially [5].) The Dirac equation for massless quark in Euclidean space time has the form:

$$
-i \gamma_{\mu} \nabla_{\mu} \psi_{n}(x)=\lambda_{n} \psi_{n}(x)
$$

where $\psi_{n}(x)$ and $\lambda_{n}$ are the eigenfunctions and eigenvalues of the Dirac operator $-\boldsymbol{\nabla}=$ $-i \gamma_{\mu} \nabla_{\mu}$. Expand the quark fields operators into the left and right ones

$$
\begin{aligned}
\psi & =\frac{1}{2}\left(1+\gamma_{5}\right) \psi_{L}+\frac{1}{2}\left(1-\gamma_{5}\right) \psi_{R} \\
\psi^{+} & =\psi_{L}^{+} \frac{1}{2}\left(1+\gamma_{5}\right)+\psi_{R}^{+} \frac{1}{2}\left(1-\gamma_{5}\right),
\end{aligned}
$$

where

$$
\gamma_{5} \psi_{L}=\psi_{L}, \quad \gamma_{5} \psi_{R}=-\psi_{R}
$$

Then for nonzero $\lambda_{n}$ the Lagrangian and the action reduces to the sum of two terms

$$
L=-\int\left[\psi_{L}^{+} \nabla \psi_{R}+\psi_{R}^{+} \nabla \psi_{L}\right] d^{4} x
$$

completely symmetric under interchange $L \longleftrightarrow R$. Therefore the solutions of the equations for left and right quark fields are also the same - the states, constracted from left and right quarks are completely symmetrical. This conclusion was obtained for fixed gluon field. It is evident, that the averaging over the gluon fields does not change it. Quite different situation arises in case $\lambda_{0}=0$. The contribution of this term to the Lagrangian:

$$
\Delta L=\int d^{4} x\left[\psi_{L}^{+}+\psi_{R}^{+}\right] \nabla \psi_{0}
$$

is equal to zero and no conclusion can be done about the symmetry of states build from left and right quark fields. One of the consequences from the said above is that all chirality violating vacuum condensates in QCD arise from zero mode solutions of Dirac equations (3).

These general arguments are supported by the well known facts:

1. The general representation of the trace of quark propagator $S(x)$ is expressed through the spectral function $\rho(\lambda)$ as a function of eigenvalues $\lambda$ (Källen-Lehmann representation):

$$
\operatorname{Tr} S\left(x^{2}\right)=\frac{1}{\pi} \int d \lambda \rho(\lambda) \Delta\left(x^{2}, \lambda\right)
$$

At $x^{2}=0 \Delta\left(x^{2}, \lambda\right)$ reduces to $\delta(\lambda)$ and we have (in Minkowski space-time):

$$
\rho(0)=-\pi\langle 0|\bar{\psi}(0) \psi(0)| 0\rangle .
$$

(The Banks-Casher relation [6]).

2. The zero-mode solution of (3) for massless quark in the instanton field is the right wave function $-\psi_{R}(x)=\left(1-\gamma_{5}\right) \psi(x)$ and in the field of anti-instanton is the left one $\psi_{L}(x)=\left(1+\gamma_{5}\right) \psi(x)[7,8]$. 
Basing on the statements, presented above, let us formulate the model for calculation of chirality violating vacuum condensates in QCD. Suppose, that vacuum expectation value (v.e.v.) of the chirality violating operator $O_{\text {c.v. }}$ is proportional to matrix element $\psi_{0}^{+} O_{\text {c.v. }} \psi_{0}$, where $\psi_{0}$ is the zero-mode solution of Eq.(3) in Euclidean space-time:

$$
\left\langle 0\left|\bar{\psi} O_{c . v .} \psi\right| 0\right\rangle \sim \psi_{0}^{+} O_{c . v .} \psi_{0} .
$$

$\psi_{0}$ depends on $x$, on the position of the center of the solution $x_{c}$, as well as on its size $\rho$ : $\psi_{0}=\psi_{0}\left(x-x_{c}, \rho\right)$. Eq.(10) must be integrated over $x_{c}$, what is equivalent to integration over $x-x_{c}$. (In what follows the notation $x$ will be used for $x-x_{c}$.) We assume, that $\rho=$ Const and find its value from comparison with the known v.e.v.'s. Finally, introduce in (10) the coefficient of proportionality $n$. So, our assumption has the form:

$$
\left\langle 0\left|\bar{\psi}(0) O_{c . v .} \psi(0)\right| 0\right\rangle=-n \int d^{4} x \psi_{0}^{+}(x, \rho) O_{c . v .} \psi_{0}(x, \rho)
$$

Our model is similar to delute instanton gas model [9], where $x_{c}$ is the position of instanton center. Unlike the latter, where the instanton density has dimension $4, n$ has dimension 3 and may be interpreted as the density of zero-modes centers in 3-dimension space. Note, that the left-hand side of (11) is written in the Minkowski space-time, while the right-hand side in Euclidean ones. (The sign minus is put in order to have $n$ positive.) In perturbative calculation in case, when the number of flavours is more then $1, N_{f}>1$, the contribution of instantons to the action is suppressed by the factor, proportional to the product of $N_{f}-1$ light quark masses. Therefore, the assumption (11) means that such suppression is absent in nonperturbative calculation. For $x$ and $\rho$-dependens of $\psi_{0}(x, \rho)$ we take the form of the zero-mode solution in the field of instanton in $S U(2)$ colour group:

$$
\psi_{0}(x, \rho)=\frac{1}{2}\left(1-\gamma_{5}\right) \frac{1}{\pi} \frac{\rho}{\left(x^{2}+\rho^{2}\right)^{3 / 2}} \chi_{0}
$$

where $\chi_{0}$ is the spin-colour isospin $(|\mathbf{T}|=1 / 2)$ wave function, corresponding to the total spin $\mathbf{I}+\mathbf{T}=\mathbf{J}$ equal to zero, $J=0 . \psi_{0}(x, \rho)$ is normalized to 1 :

$$
\int d^{4} x \psi^{+}(x, \rho) \psi(x, \rho)=1
$$

Consider first the quark condensate $\langle 0|\bar{q} q| 0\rangle$, the most important chirality violating v.e.v., determining the values of baryon masses [1]-[11]. (Here $q=u, d$ are the fields of $u, d$-quarks). In this case $O_{c . v}=1$ and in accord with $(11),(13)$ we have

$$
n=-\langle 0|\bar{q} q| 0\rangle=(1.65 \pm 0.15) \times 10^{-2} \mathrm{GeV}^{3}(\text { at } 1 \mathrm{GeV})[10]
$$

(The integration over $S U(2)$ subgroup position in $S U(3)$ colour group as well as antiinstanton contribution are included in the definition of $n$.) The anomous dimension of quark condensate is equal to 4/9. According to (14) $n$ has the same anomalous dimension. The size $\rho$ of the zero-mode wave function can be found by calculation in the framework of our model of the v.e.v.

$$
-g\left\langle 0\left|\bar{\psi} \sigma_{\mu \nu} \frac{\lambda^{n}}{2} G_{\mu \nu}^{n} \psi\right| 0\right\rangle \equiv m_{0}^{2}\langle 0|\bar{q} q| 0\rangle
$$


The parameter $m_{0}^{2}$ is equal to [13]: $m_{0}^{2}=0.8 \mathrm{GeV}^{2}$ at $1 \mathrm{GeV}$. The $m_{0}^{2}$ anomalous dimension is equal to $-14 / 27$. Working in the $S U(2)$ colour group, substitute $\lambda^{n}$ by $\tau^{a}(a=1,2,3)$ and take for $G_{\mu \nu}^{a}$ the instanton field

$$
G_{\mu \nu}^{a}(x, \rho)=\frac{4}{g} \eta_{a \mu \nu} \frac{\rho^{2}}{\left(x^{2}+\rho^{2}\right)^{2}},
$$

where the parameter $\eta_{a \mu \nu}$ were defined by 't Hooft [14] (see also [5]). The substitution of (12) and (16) into (11) gives after simple algebra

$$
\frac{1}{2} n \frac{1}{\rho^{2}}=m_{0}^{2} n
$$

Therefore,

$$
\rho=\frac{1}{\sqrt{2} m_{0}}=0.79 \mathrm{GeV}^{-1}=0.256 \mathrm{fm}(\text { at } 1 \mathrm{GeV}) .
$$

This value of $\rho$ is close to ones, used in the delute instanton gas or instanton liquid models.

We are now in a position to calculate less well known quantities - the magnetic susceptibilities of quark condensate, induced by external constant electromagnetic field.

The dimension 3 quark condensate magnetic susceptibility is defined by [15]:

$$
\left\langle 0\left|\bar{q} \sigma_{\mu \nu} q\right| 0\right\rangle_{F}=e_{q} \chi\langle 0|\bar{q} q| 0\rangle F_{\mu \nu}, \quad q=u, d,
$$

where quarks are considered as moving in external constant weak electromagnetic field $F_{\mu \nu}$ and $e_{q}$ is the charge of quark $q$ in units of proton charge (the proton charge $e$ is included in the definition of $F_{\mu \nu}$ ). The left-hand side of (19) violates chirality, so it is convenient to separate explicitly the factor $\langle 0|\bar{q} q| 0\rangle$ in the right-hand side. It was demonstrated in [15] that $\left\langle 0\left|\bar{q} \sigma_{\mu \nu} q\right| 0\right\rangle_{F}$ is proportional to the charge $e_{q}$ of the quark $q$. A universal constant $\chi$ is called the quark condensate magnetic susceptibility.

Let us determine the value of $\chi$ in our approach. For this goal it is necessary to consider Eq.3 in the presence of external constant electromagnetic field $F_{\mu \nu}$ and to find the first order in $F_{\mu \nu}$ correction to zero mode solution (12). This can be easily done by representing $\psi$ as

$$
\psi(x, \rho)=\psi_{0}(x, \rho)+\psi_{1}(x, \rho),
$$

where $\psi_{0}$ is given by $(12)$ and $\psi_{1}$ represents the proportional to $F_{\mu \nu}$ correction. Substitute (20) in Eq.3 added by the term of interaction with electromagnetic field, neglect $\psi_{1}$ in this term and solve the remaining equation for $\left.\psi_{1}(x, \rho)\right)$. The result is:

$$
\psi_{1}(x, \rho)=\frac{1}{16} e_{q} \eta_{a \mu \nu} \sigma_{a} F_{\mu \nu} x^{2}\left(1+\frac{1}{2} \frac{x^{2}}{\rho^{2}}\right) \psi_{0}(x, \rho)
$$

where $\sigma_{a}$ are Pauli matrices. The matrix element $\psi^{+} \sigma_{\mu \nu} \psi$ appears to be equal:

$$
\psi^{+} \sigma_{\mu \nu} \psi=-\frac{1}{2} e_{q} F_{\mu \nu} \psi_{0}^{+} x^{2}\left(1+\frac{1}{2} \frac{x^{2}}{\rho^{2}}\right) \psi_{0}
$$

(The properties of $\eta_{a \mu \nu}$ symbols [14],[5] were exploited.) The v.e.v. (19) in the Minkowski space-time is given by:

$$
\left\langle 0\left|\bar{\psi} \sigma_{\mu \nu} \psi\right| 0\right\rangle_{F}=e_{q} F_{\mu \nu} n \frac{1}{\pi^{2}} \int d^{4} x x^{2}\left(1+\frac{1}{2} \frac{x^{2}}{\rho^{2}}\right) \frac{\rho^{2}}{\left(x^{2}+\rho^{2}\right)^{3}}
$$


(The normalization condition (13) for $\psi_{0}(x, \rho)$ was used.) It is convenient to express $n$ through quark condensate by (14), use the notation $x^{2}=r^{2}$, where $r$ is the radius-vector in 4-dimensional space. Then according to (19) we have:

$$
\chi=-\rho^{2} \int_{0}^{R^{2}} d r^{2} r^{4}\left(1+\frac{1}{2} \frac{r^{2}}{\rho^{2}}\right) \frac{1}{\left(r^{2}+\rho^{2}\right)^{3}}
$$

The integral (24) is quadratically divergent at large $r$. So, the cut-off $R$ is introduced. Its value can be estimated in following way. The volume occupied by one zero-mode in 3dimensional space is approximately equal to $1 / n$ (the volume of the Wigner-Seitz cell). So, for cut-off radius square $R^{2}$ in four-dimensions we put

$$
R^{2}=\frac{4}{3}\left(\frac{3}{4 \pi n}\right)^{2 / 3}=7.92 \mathrm{GeV}^{-2}
$$

where the factor $4 / 3$ corresponds to transition from 3 to 4 dimensions. The calculation of the integral (24) at the values of parameters $\rho(18)$ and $R^{2}(25)$ gives

$$
\chi=-3.52 \mathrm{GeV}^{-2}
$$

The quark condensate magnetic susceptibility was previously calculated by QCD sum rule method [16]-[19] and expressed through the masses and coupling constants of mesonic resonances. The recent results are:

$$
\chi(1 \mathrm{GeV})=-3.15 \pm 0.3 \mathrm{GeV}^{-2}[18] ; \quad \chi(1 \mathrm{GeV})=-2.85 \pm 0.5 \mathrm{GeV}^{-2}[19]
$$

(The earlier results, obtained by the same method, were: $\chi(0.5 \mathrm{GeV})=-5.7 \mathrm{GeV}^{-2}[16]$ and $\chi(1 \mathrm{GeV})=$

$-4.4 \pm 0.4 \mathrm{GeV}^{-2}[17]$.) The anomalous dimension of $\chi$ is equal to $-16 / 27$. It was accounted in [16]-[19], but not in the presented above calculation. (In some of these papers, the $\alpha_{s^{-}}$ corrections and continuum contribution, were also accounted.) One can believe, that the value (26) refer to $1 \mathrm{GeV}$, because the value of quark condensate (14) refer to this scale and also because the scale $1 \mathrm{GeV}$ is a typical scale, where, on the one hand, the zero-modes and quark condensates are quite important (see, e.g. [12]) and, on the other, the instanton gas model is valid [9]. Since the integral is quadratically divergent it is hard to estimate the accuracy of (26). I guess, that it is not worse, than 30-50\%. In the limit of this error the result (26) is in an agreement with those found in phenomenological approaches.

Turn now to quark condensate magnetic susceptibilities of dimension $5, \kappa$ and $\xi$ defined in Ref.[15]

$$
\begin{gathered}
g\left\langle 0\left|\bar{q} \frac{1}{2} \lambda^{n} G_{\mu \nu}^{n} \bar{q}\right| 0\right\rangle_{F}=e_{q} \kappa F_{\mu \nu}\langle 0|\bar{q} q| 0\rangle, \\
-i g \varepsilon_{\mu \nu \rho \tau}\left\langle 0\left|\bar{q} \gamma_{5} \frac{1}{2} \lambda^{n} G_{\rho \tau}^{n} q\right| 0\right\rangle_{F}=e_{q} \xi F_{\mu \nu}\langle 0|\bar{q} q| 0\rangle
\end{gathered}
$$

Perform first the calculation of $\kappa$. In this case the expression of $\psi_{1}(x, \rho)(21)$ must be multiplyed by the additional factor: $\frac{1}{2} \tau^{b} G_{\mu \nu}^{b}$ where $G_{\mu \nu}^{b}$ is given by (16) and the indices $\mu, \nu$ in (21) are changed to $\lambda, \sigma$. In the further calculation it will be taken into account, that $\chi_{0}$ in (12) corresponds to total spin-colour isospin $J=0$ and consequently

$$
\boldsymbol{\sigma} \tau \chi_{0}=-3 \chi_{0}, \quad \sigma^{a} \tau^{b} \chi_{0}=-\delta^{a b} \chi_{0}
$$


In the relation

$$
\eta_{b \mu \nu} \eta_{b \lambda \sigma}=\delta_{\mu \lambda} \delta_{\nu \sigma}-\delta_{\mu \sigma} \delta_{\nu \lambda}+\varepsilon_{\mu \nu \lambda \sigma}
$$

the last term drops out after summation of zero-modes from instanton and anti-instanton configuration. The final result for $\kappa$ is:

$$
\kappa=-\int_{0}^{z} u^{2} d u \frac{1}{(u+1)^{4}}\left(1+\frac{1}{2} u\right)=-\frac{1}{2}\left[\ln (z+1)-\frac{13}{6}+\frac{1}{z+1}+\frac{1}{2} \frac{1}{(z+1)^{2}}-\frac{1}{3} \frac{1}{(z+1)^{3}}\right],
$$

where $z=R^{2} / \rho^{2}=12.7$. Numerically, we have:

$$
\kappa=-0.26
$$

The calculation of $\xi$ is very similar to those of $\kappa$ and the result is

$$
\xi=2 \kappa=-0.52
$$

The values of $\kappa$ and $\xi$ only logarithmically depend on the cut-off. But unfortunately the logarithm in (32) is not very large and its main part is compensated by the term $-13 / 6$, appearing in (32). So, the accuracy of (33),(34) can be estimated as about $30 \%$. The phenomenological determination of 5 -dimensional quark condensate magnetic susceptibilities was performed by Kogan and Wyler [20] along the same lines, as it was done in [16],[17]. No anomalous dimensions were accounted. The results of [20] are:

$$
\kappa=-0.34 \pm 0.1, \quad \xi=-0.74 \pm 0.2
$$

As can be seen, they are in a good agreement with (33),(34). The 5-dimensional quark condensate magnetic susceptibilities play a remarkable role in determination of $\Lambda$-hyperon magnetic moment [21].

I conclude this part of my talk. It was argued, that chiral symmetry violation in QCD arises due to zero-mode solution of Dirac equation for massless quark in arbitrary gluon field. The model is proposed similar to delute instanton gas model, in which the zero-mode solution is the same as in the field of instanton. The parameters of the model: the density of zeromodes and their size are determined from the values of quark and quark-gluon condensates. In the framework of this model the values of quark condensates magnetic susceptibilities of dimensions 3 and 5 were calculated in agreement with ones found by QCD sum rules method using the properties of hadronic spectrum. The agreement of these two approaches gives the strong argument in favour that the instantons are the only source of chirality violating condensates in QCD.

Turn now to the temperature dependence of quark condensate, considered as an order parameter. It is expected that quark condensate vanishes at high temperatures and the chiral symmetry is restored. Two possibilities are discussed: the second order phase transition and the crossover. In our approach the normalization condition (13) takes place at any temperature [22]. So, the temperature dependence of quark condensate reduces to

$$
\alpha(T) \equiv\langle 0|\bar{q} q|\rangle_{T}=n(T)
$$

One may expect that $n(T)$ vanishes only in the case, when the quantum number, curried by instanton is vanishing. This quantum number is the topological charge, which is temperature 
independent. So, in our approach $n(T)$ never vanishes and the phase transition is of the type of crossover. The order parameter temperature dependence of the second order phase transition near the critical point $T_{c}$ is smeared by fluctuations [23] (The same statement

refers, surely, to the crossover.) The fluctuation are determined by long wave oscillations of the fields. In QCD they are given by small frequency gluonic field $G_{\mu \nu}^{n}$, which is a constant. We have:

$$
\frac{\Delta \alpha\left(T_{c}\right)}{\alpha\left(T_{c}\right)} \sim\left\langle 0\left|G_{\mu \nu}^{2}\right| 0\right\rangle T_{c}^{2} \rho^{6}
$$

where $\Delta \alpha\left(T_{c}\right)$ is of the order of magnitude of the variation of $\alpha(T)$ near the crossover critical point. It is evident, that the left hand side should vanish, if $T_{c}$ would be zero. I guess, that the factor, corresponding to this circumstance is $T_{c}^{2}$. The factor $\rho^{6}$ is added for dimensional reasons.

All the discussion of the $\alpha(T)$ temperature dependence is based on implicit assumption, that this object has a physical meaning at finite $T$. It is unclear, however, how it can be defined. I did not succeed to formulate the gedanken-experiments, in which this object could be measured at finite $T$. Therefore, may be, such an object has no physical sence at $T \neq 0$.

This work is supported by RFBR grant 09-02-00732 and in part by CRDF Cooperative Program grant RUP2-2961-MO-09. I acknowledge the support of the European CommunityResearch Infrastructure Integrating Activity "Study of Strongly Interacting Matter" under the Seventh Framework Program of EU.

\section{References}

[1] B.L.Ioffe, Nucl. Phys. B188, 317 (1981), Errata, ibid B192, 591 (1982).

[2] B.L.Ioffe, Phys.Lett. B678, 512 (2009).

[3] B.L.Ioffe, Usp.Fiz.Nauk 176 (2008) 1103, Phys.Usp. 49, 1077 (2006).

[4] Instantons in Gauge Theories, ed. by M.Shifman, World Sci., 1994.

[5] A.I.Vainshtein, V.I.Zakharov, V.A.Novikov and M.A.Shifman, Usp. Fiz. Nauk 136, 553 (1982) (Sov. Phys. Uspekhi 25, 195 (1982)), Reproduced in [1], p.468.

[6] T.Banks and A.Casher, Nucl. Phys. B169, 103 (1980).

[7] L.S.Brown, R.Carlitz and C.Lee, Phys. Rev. D16, 417 (1977).

[8] R.Jackiw and C.Rebbi, Phys. Rev. D16, 1052 (1977).

[9] T.Schäfer and E.V.Shuryak, Rev. Mod. Phys. 70, 323 (1998).

[10] B.L.Ioffe, Zs. Phys. C18, 67 (1983).

[11] B.L.Ioffe, ArXiv: 0810.4234, Phys. At. Nucl. 72, 1214 (2009).

[12] B.L.Ioffe, Prog. Part. Nucl. Phys. 56, 232 (2006).

[13] V.M.Belyaev and B.L.Ioffe, Sov. Phys. JETP 56, 493 (1982).

[14] G. 't Hooft, Phys. Rev. D16, 3432 (1976). 
[15] B.L.Ioffe and A.V.Smilga, Nucl. Phys. B232, 109 (1984).

[16] V.M.Belyaev and I.I.Kogan, Yad. Fiz. 40, 1035 (1984).

[17] I.I.Balitsky, A.V.Kolesnichenko and A.V.Yung, Sov. J. Nucl. Phys. 41, 138 (1985).

[18] P.Ball, V.M.Braun and N.Kivel, Nucl. Phys. B649, 263 (2003).

[19] J.Rohrwild, JHEP 0709:073 (2007).

[20] I.I.Kogan and D.Wyler, Phys. Lett. B274, 100 (1992).

[21] J.Pasupathy, J.P.Singh, S.L.Wilson and C.B.Chiu, Phys. Rev. D36, 1442 (1987).

[22] D.J.Gross, R.D.Pisarski and L.G.Yaffe, Rev.Mod.Phys. 53 (1981) 43.

[23] L.D.Landau and E.M.Lifshitz, Statistical Physics, Part I, Pergamon Press, 1980. 